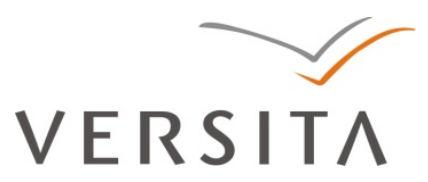

BULGARIAN ACADEMY OF SCIENCES

CYBERNETICS AND INFORMATION TECHNOLOGIES • Volume 13, Special Issue

Sofia $\bullet 2013$

Print ISSN: 1311-9702; Online ISSN: 1314-4081

DOI: $10.2478 /$ cait-2013-0040

\title{
Distributed Energy Balance Clustering Algorithm in Wireless Sensor Networks
}

\section{Yang Wang}

School of Electronics and Communication Engineering, Shenzhen Polytechnic, Shenzhen, China Email: 751559821@qq.com

\begin{abstract}
The traditional clustering algorithm of Low-Energy Adaptive Clustering Hierarchy (LEACH) does not care about the residual energy of the cluster heads and the distance relationship of each node in the wireless sensor networks (WSNs). Each new round the cluster head is changed without any concern about the residual energy of the current cluster head, and the nodes join into a new cluster head without concern about the distance factor and the residual energy factor of all nodes in WSNs. To solve these problems in LEACH, this paper puts forward a distributed energy balance clustering algorithm, which takes into full consideration the residual energy of the cluster heads and the distance relationship between the base station and the cluster heads. The experimental results indicate that the proposed distributed energy balance clustering algorithm can prolong the lifetime of the networks more than LEACH, and the number of messages for selecting a new cluster head at the same time can be greatly reduced, which proves it as more suitable for practical applications.
\end{abstract}

Keywords: Wireless sensor networks, Low-Energy Adaptive Clustering Hierarchy, residual energy, distance relationship, distributed energy balance clustering algorithm.

\section{Introduction}

In Ad Hoc, the primary goals of the wireless networks are to provide high quality services, fair and efficient use of the network bandwidth [1,2]. The evaluation 
standard of the routing protocols includes validity, robustness, fairness, strategy, a small delay and energy consumption. Wireless Sensor Networks (WSNs) collect and process information by a multi-hop wireless communication method, which can be sent to Internet [3,4]. Because of the energy limit of WSNs, the concept to prolong the life period of WSNs as long as possible is the most important problem $[5,6]$ in practical applications. Also, considering the energy limit of WSN, the traditional Ad Hoc network clustering algorithm cannot be directly applied to WSNs. In different application surroundings, the most suitable routing protocol of WSNs might be different. Compared with the traditional routing protocols, the routing protocols of WSNs must have the following characteristics:

- Energy point: the traditional routing protocols select the optimal path without any concern about the energy consumption factor. But the energy of WSNs is limited, and the power supply is usually hard to replace. In order to realize the load sharing of the whole network and prolong the life cycle of the network, the energy problem must be considered [7].

- Data point: the traditional routing protocols usually use an address for the selection identification of the route, each node has a unique IP address as a network identifier. But the nodes of WSNs may be placed randomly, concerning only about the sensor data in the monitor area. In a large scale of WSN, there must be a large number of nodes' IP addresses, which may require more time to transmit the IP data than the real needed data, so that the routing protocols must pay more attention to the data communication mode and flow direction [8].

- Application point: there are many kinds of WSNs' applications, so it is impossible to find out one routing mechanism suitable for all applications. In every application, the data communication function may be different from the others, so every application must design a specific routing mechanism suitable for its own [9].

According to the properties of different WSNs' applications, there are several kinds of routing protocols studied: routing protocols based on data dealing, routing protocols based on clusters, routing protocols based on the location, routing protocols based on the data flow model and the requirements of service quality, and so on.

The main routing protocols based on data include Flooding Protocol, Gossiping Protocol and Sensor Protocol for Information via Negotiation (SPIN), and Directed Diffusion Protocol. Flooding Protocol is a kind of traditional routing technologies, based on the traditional wired network flooding routing protocol. The Flooding Protocol does not require routing computation, the node receives a message and then broadcasts data packets to all adjacent nodes, and repeats again, until the data packets reach the destination or the preset maximum hop count has been achieved. Flood route is easy to achieve, but its energy efficiency is very poor, and may lead to an implosion and overlap of the messages. To solve these problems, Gossiping Protocol was proposed to adopt the random principle, which can prevent message implosion [10]. SPIN adopts the consultation rules among the nodes and the source by an adaptive mechanism solving the flooding problems [11].

There are routing protocols based on geographical location information that send data to the target node through position information and without broadcast 
finds out the target node. These main protocols include the Geographical and Energy Aware Routing (GEAR) and Graph Embedding (GEM) protocols based on geographical location information. GEAR protocol is based on the event region location information to find the optimal path from the sink node to the event region, which can avoid flooding problems and reduce the cost of route establishment [12]. GEM protocol is a geographic routing protocol suitable for storage mode of the data center, whose basic idea is to build a Virtual Polar Coordinate System (VPCS) to represent the actual network topology [13].

The routing protocols which are based on a data stream model can satisfy the communication requirements of the service quality when supporting a data routing service. Some of these protocols select the data path by computing the nodes' left energy, and how much energy is required to send a message to prolong the lifetime of the network. Typical protocols are the Reliable Information Forwarding Using Multiple paths (ReInForM) and the SPEED protocol. ReInForM considers the reliability requirement, the channel quality and the jump number from a source node to a destination node, and decides the number of the transmission paths and the next hop, which can satisfy the reliability requirement of data transmission [14]. The SPEED protocol is a real-time routing protocol, which can realize the point-topoint transmission rate guaranteed, network congestion control and load balancing mechanism [15].

The routing protocols based on clusters divide WSNs' nodes into different clusters, and each cluster has one cluster head. In a cluster, every node sends its data to the cluster head firstly, and then the cluster head forwards the data to the base station, in this way the network traffic can be reduced very much. The typical protocols include Low-Energy Adaptive Clustering Hierarchy (LEACH) [16], Power-Efficient Gathering in Sensor Information Systems (PEGASIS) [17], and Thresh-old Sensitive Energy Efficient Sensor Network Protocol (TEEN) [18], and others $[19,20]$.

The earliest cluster routing protocol proposed is LEACH, which is the base of many cluster-based routing protocols, such as TEEN and Hybrid Energy-Efficient Distributed Clustering (HEED). The LEACH basic idea is to divide the nodes into different clusters appropriately first, and then to select cluster heads in each cluster. In one cluster the nodes communicate directly with the cluster head, after the cluster head receives all data from the cluster, the cluster head begins data fusion and then sends the result to the sink node. LEACH selects a cluster head randomly and tries to share relay communication business averagely to achieve the network load sharing uniformly. The "round" concept is defined by LEACH - in each round LEACH selects again randomly a cluster head. After being selected, the cluster head undertakes relay communication business. In the next round, LEACH selects a cluster head and clusters again. LEACH can prolong the lifetime of the network by distributing the whole network energy load to each node in a cluster [21, 22].

PEGASIS is developed by LEACH. In PEGASIS the network is assumed as composed of isomorphism static or slow-moving nodes. Firstly the node sends a test signal under lower radio power, and then detects the response to determine the nearest neighbor node. In this way all nodes in the network can define each other's 
location, and then select the cluster according to their location, which can help the cluster head optimize the best route to the destination through a reference location. TEEN adopts the clustering method which is similar to LEACH, but TEEN uses different strategies during the process of transferring data. In LEACH the nodes keep monitoring the environment and send data to the cluster head in each round during the assigned timeslot, that is called active network. In contrast, TEEN is a responsive network, a node sends data only when the observed object has a sudden or drastic change.

This paper focuses on the routing protocol based on clusters. However, few studies have been done on the energy and the distance factors in a previous research which should be applied to LEACH algorithm. In practice, the residual energy of every node maybe very different after a period of time running, which means the cluster head may be still the node with the largest residual energy in the whole cluster. Furthermore, the distance factor should also be considered into LEACH algorithm. Generally, the closer the cluster heads are to the base station, the more energy will be consumed, because of forwarding more messages from the other nodes. So, according to the distance from the base station, the scale of the cluster must be different. If the distance is closer, the scale of the cluster would be smaller; on the contrary, if the distance is long, the scale of the cluster would be larger. How to apply the distance factor into the LEACH algorithm is worth studying.

However, in order to prolong the lifetime of the network through energy balancing, more research should focus on balancing the energy consumed by the cluster members. On this basis, this paper puts forward one improved distributed energy balance clustering algorithm which pays more attention to energy consuming among the cluster heads. Firstly, the cluster head selecting mechanism is improved in this paper, which takes the residual energy of every node in the cluster, including the cluster head itself into account. Thus the residual energy factor of a node can be used to balance the energy cost of the whole cluster. Secondly, the distance factor is used to help the cluster heads adjust their wireless transmission power, which can change the scale of a cluster. Because the cluster head is closer to the base station, more energy is consumed to forward data to the base station. Using the distance factor to adjust the nodes number of a cluster helps the cluster head decrease the energy consumed by the internal cluster data forwarding. More energy will remain to forward data among the cluster heads in a multi hop network, which can balance the energy cost of the whole network. Thirdly, this paper uses simulation and actual measurement to test and verify the improved algorithm. Through simulation it gets the proper parameter value. Then, in the actual measurement, this paper adopts $100 \mathrm{WSN}$ nodes to test and verify the result.

\section{Algorithm design}

For the distributed energy balance clustering protocol, during the cluster setting period, every cluster node produces one random number between " 0 " and " 1 "; if the number is smaller than the threshold value, the node will become the next candidate cluster head. Through the competition mechanism, the candidate cluster head will 
become the cluster center, and this center broadcasts the message including the cluster head information to all the nodes around itself. Each node in WSN can decide which cluster head to join on the basis of the algorithm proposed in this paper and the response of the cluster head. During the data transmission period, each node in a cluster sends data to the cluster head according to TDMA protocol. After the cluster head finishes data fusion it sends a message to the network gate. After the pre-set period, the cluster head will be selected again by the protocol.

How to select the cluster head is the key of the cluster foundation. The traditional LEACH algorithm does not consider the residual energy and the distance factor. The threshold value function $T(n)$ is given by

$$
T_{(n)}=\left\{\begin{array}{c}
\frac{k}{N-k \times[r \cdot \bmod (n / k)]}, n \in G, \\
0, \quad n \notin G,
\end{array}\right.
$$

where $N$ is the nodes number of the whole network, $k$ is the cluster heads number of the whole network, $r$ is the finished rounds number, and $G$ is the set of nodes which are not selected for the cluster head during last $r$ round times. According to (1), the traditional LEACH algorithm only cares about the cluster heads number and the rounds number, which cannot reflect the residual energy of each node. After a period of time running, the residual energy of every node maybe very different, that means the cluster head may be still the node with the biggest residual energy in the whole cluster.

To solve the drawbacks, this paper has improved the threshold value function $T_{e}(n)$ as given by

$$
T_{e}(n)=\left\{\begin{array}{c}
\frac{p}{1-p \times[r \cdot \bmod (1 / p)]} \cdot E_{f}(n), n \in M, \\
T_{c}(E(n)), \quad n \notin M,
\end{array}\right.
$$

where $T_{e}(n)$ is the threshold function for every node in the cluster, $p$ is the percent of cluster heads to all nodes, $r$ is the rounds number selected, $n$ is the nodes number, $M$ is the set of nodes that are not selected for cluster heads during the last $1 / p$ rounds, and $E(n)$ is the residual energy of node $n . T_{c}(n)$ is used as the threshold value function of the cluster head and is given by

$$
T_{c}(n)= \begin{cases}0 & E(n)<E_{\text {bigest }}, \\ 1, & E(n) \geq E_{\text {bigest }},\end{cases}
$$

where $E_{\text {bigest }}$ is the remaining energy maximum value of the whole net. $E_{f}(n)$ is the rate of the energy left at node $n$ to $E_{\text {total }}$ and given by

$$
E_{f}(n)=\frac{E(n)}{E_{\text {total }}},
$$

where $E_{\text {total }}$ is the total residual energy of the whole net. The threshold value function proposed by this paper gives the current cluster head the opportunity to continue to be the head, connected with the residual energy of every node. In fact, when a new round begins, if the current cluster head is still the node with the 
maximum residual energy in the whole cluster, it can remain the cluster head, which means that information transmission during the cluster head selecting can be reduced very much.

Except the energy factor, the scale of cluster formation must also consider the distance between the cluster head and the base station. During the initial process of network establishment, the base station broadcasts the standard message by the full radio power to the whole WSN. All nodes receive the message as a signal to calculate the received signal strength indication, which can reflect indirectly the distance from the base station. Generally, the closer the cluster heads are to the base station, the more energy will be consumed because of forwarding more messages from other nodes to the base station. Thus according to the distance from the base station, the scale of the cluster would be different. If the distance is closer, the scale of the cluster would be smaller; on the contrary, if the distance is longer, the scale of the cluster would be larger.

If the node becomes a cluster head, the number of the cluster nodes must satisfy

$$
N_{b}(n)= \begin{cases}\operatorname{Num}_{\text {org }} \times(1+u), & w \geq 0.5, \\ \operatorname{Num}_{\text {org }} \times(1-v), & w<0.5,\end{cases}
$$

where $N_{b}(n)$ is the number of cluster nodes which the new cluster head can manage, and $\mathrm{Num}_{\text {org }}$ is the original number of the last cluster head included. The variables $u$ and $v$ are the independent scale factor, which can be adjusted based on the application. And $w$ is given by

$$
w=\frac{D(n)-d_{\min }}{d_{\max }-d_{\min }}\left(\frac{1}{p}-1\right),
$$

$D(n)$ is the distance of the cluster head $n$ from the base station, $d_{\max }$ is the maximum distance from the base station, and $d_{\min }$ is the minimum distance from the base station, $p$ is the percent of the cluster heads to all nodes. If $w \geq 0.5$, the node number of the new cluster head must be increased by $1+u$. But if $w<0.5$, the node number of the new cluster head must be decreased by $1-v$. This function can ensure that the cluster head which is closer to the base station can manage a smaller number of nodes, which can decrease the energy consumed in internal data forwarding of the cluster as much as possible.

To adjust the number of nodes that the cluster head has, we must set the coverage radius of the cluster head. With the distance between a cluster head and the base station decreasing, the coverage radius of the cluster head must decrease accordingly. For example, radius $R 1$ must be the smallest and radius $R 3$ - the biggest in Fig. 1. In order to control the cluster radius, the cluster head must adjust their radio transmitting power according to the distance $D(n)$. The radio transmitting power $T_{x}(n)$ is given as

$$
T_{x}(n)=\varepsilon_{\mathrm{rf}}\left(\frac{D(n)-d_{\min }}{d_{\max }-d_{\min }}\right) \cdot T_{\max },
$$




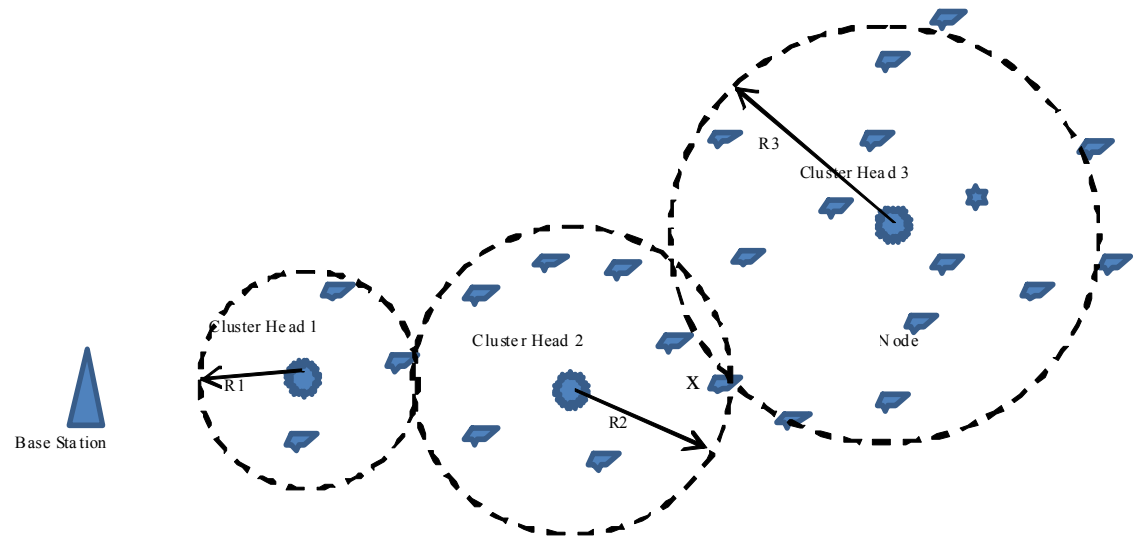

Fig.1. The different coverage radius of different cluster head

where $T_{\max }$ is the maximum transmitting power value of node $n$, and $\varepsilon_{\mathrm{rf}}$ is the coefficient, whose value range is $(0,2)$. In the applications, the nodes must adjust the value of $\varepsilon_{\mathrm{rf}}$ to satisfy the radius change. Thus, if the WSNs' nodes can adjust the radio transmitting power, it is easy to control the number of the cluster nodes. But if WSNs' nodes cannot adjust the radio transmitting power, the number of the cluster nodes must be controlled by the protocol.

After the cluster head is selected, how the nodes join into the cluster head is very important, and it decides the energy consuming balance of the cluster coverage area. For example, in Fig. 1, node $x$ must choose one cluster head to join. Through observation, node $x$ can join cluster head 2 or cluster head 3. In fact, node $x$ is closer to cluster head 2 than to cluster head 3, but it must not join cluster head 2 directly only because of the distance factor.

To select a cluster head to join, the distance must not be the only factor considered by the node. This paper proposed one energy consuming function based on Distribute Energy Balance Clustering Algorithm (DEBCA) expressed as

$$
E_{\text {cons }}(n, m)=\alpha \times \eta(n, m) \times E_{n}+\beta \times \theta(m B a s e S t a t i o n) \times E_{m},
$$

where $E_{\text {cons }}(n, m)$ is the energy consuming factor function of node $n$ when cluster head $m$ is selected, and $\alpha$ is the proportional coefficient; $\eta(n, m)$ is the function of the calculated distance factor between node $n$ and cluster head $m ; \beta$ is the proportional coefficient; $\theta$ (mBaseStation) is the function of the calculated distance factor between the base station and cluster head $m ; E_{n}$ is the residual energy of node $n, E_{m}$ is the residual energy of cluster head $m$.

Considering the contribution to $E_{\text {cons }}(n, m)$, define the function $\eta(n, m)$ as

$$
\eta(n, m)=\frac{D_{p 2 p}(n, m)}{D_{m-\max }},
$$

where $D_{p 2 p}(n, m)$ is the distance between node $n$ and cluster head $m, D_{m-\max }$ is the maximum distance of all the nodes away from the cluster head $m$. And define the $\theta$ (mBaseStation) function as 


$$
\theta(m, \text { BaseStation })=\frac{D_{p 2 p} m, \text { BaseStation }}{D_{\text {BaseStation }- \text { max }}},
$$

where $D_{p 2 p}(m$, BaseStation) is the distance between cluster head $m$ and the base station, and $D_{\text {Basestation-max }}$ is the maximum distance of all cluster heads with respect to the base station. When the node wants to select one cluster head to join, it must consider the energy consuming balance to prolong the lifetime of the whole network. The rule to select a cluster head is to ensure that the energy consuming factor $E_{\text {cons }}(n, m)$ is minimal.

\section{Experimental results}

In this section we use simulation and actual measurement to verify the improved DEBCA algorithm. Firstly, we use the simulation to get the proper parameter value. Then in the actual measurement, we use 100 WSN nodes to test and verify the result.

In simulation, we use the same wireless channel energy consuming model as in [23],

$$
E_{\mathrm{tx}}(L, d)=\left\{\begin{array}{ll}
L E_{\text {elect }}+L \varepsilon_{\mathrm{fs}} d^{2}, & d<d_{0}, \\
L E_{\text {elect }}+L \varepsilon_{\mathrm{mp}} d^{4}, & d \geq d_{0},
\end{array},\right.
$$

where $L$ is the number of bits the node is transmitting, and $d$ is the distance to the target; $E_{\mathrm{tx}}(L, d)$ is the energy of transmitting circuit consuming and power amplifier consuming, and $E_{\text {elect }}$ is the energy of transmitting circuit consuming. The transmission distance $d$ determines which kind of models to use for $E_{\mathrm{tx}}(L, d), d_{0}$ is the threshold value of the distance, $\varepsilon_{\mathrm{fs}}$ and $\varepsilon_{\mathrm{mp}}$ show the energy needed in different models.

The nodes consumed energy $E_{\mathrm{rx}}(L)$ when receiving $L$ bits is

$$
E_{\mathrm{rx}}(L)=L E_{\text {elect }} \text {. }
$$

Define the simulation parameters as presented in Table 1.

Table 1. Simulation parameters

\begin{tabular}{|l|l|}
\hline \multicolumn{1}{|c|}{ Parameters } & \multicolumn{1}{c|}{ Value } \\
\hline Network coverage area & $(0,0) \sim(200,200) \mathrm{m}$ \\
\hline Location of base station & $(200,300) \mathrm{m}$ \\
\hline Nodes number & 100 \\
\hline Cluster heads number & {$[3,6]$} \\
\hline Initial energy & $0.8 \mathrm{~J}$ \\
\hline$E_{\text {elect }}$ & $45 \mathrm{~nJ} / \mathrm{b}$ \\
\hline$\varepsilon_{\mathrm{fs}}$ & $10 \mathrm{pJ} / \mathrm{b}$ per $\mathrm{m}^{2}$ \\
\hline$\varepsilon_{\mathrm{mp}}$ & $0.0013 \mathrm{pJ} / \mathrm{b}$ per $\mathrm{m}^{4}$ \\
\hline$d_{0}$ & $100 \mathrm{~m}$ \\
\hline Packet length & $500 \mathrm{bits}$ \\
\hline
\end{tabular}


Firstly, the proportional coefficient parameter $\alpha$ and $\beta$ must be determined in (8). Assuming that $\alpha \in[0,1]$ and $\beta=1-\alpha$, we simulate the algorithm in MATLAB, the result is shown in Fig. 2; when $\alpha$ is between 0.65 and 0.75 , the lifetime of the network is longer.

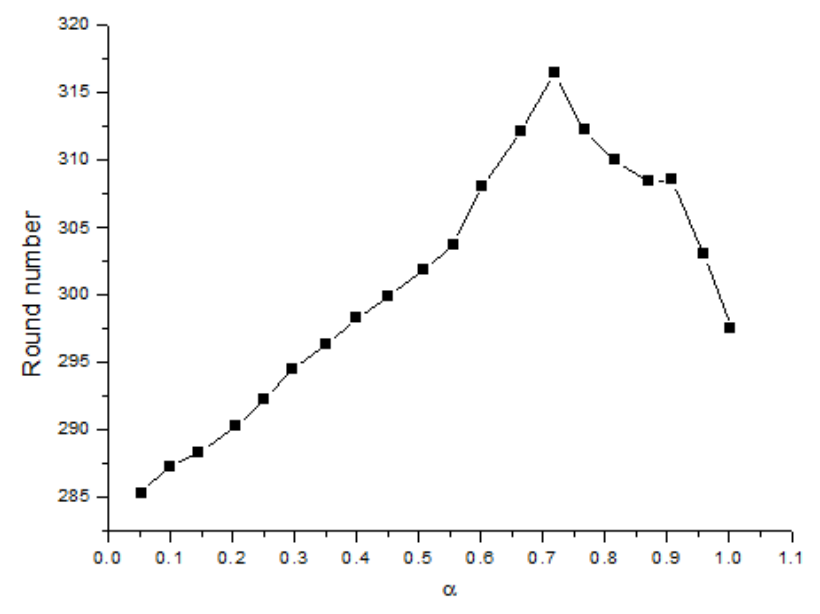

Fig. 2. The relationship between the round number $\alpha$

After that we use 100 WSN nodes to verify the result, and the related parameters are given in Table 2 .

Table 2. Actual measurement parameters
\begin{tabular}{|l|l|}
\hline \multicolumn{1}{|c|}{ Parameters } & \multicolumn{1}{c|}{ Value } \\
\hline Network coverage area & $(0,0) \sim(300,300) \mathrm{m}$ \\
\hline Location of base station & $(200,300) \mathrm{m}$ \\
\hline Nodes number & 100 \\
\hline Cluster heads number & {$[3,6]$} \\
\hline Energy support & $300 \mathrm{~mA} . \mathrm{h}$ \\
\hline Packet length & 500 bits \\
\hline$\alpha$ & 0.7 \\
\hline
\end{tabular}

In actual measurement, the 100 nodes will be located by the rule indicated in Fig. 3. They are supported by new batteries with about $300 \mathrm{~mA}$.h at each time beginning. After that the network begins to work until the network is down without any batteries changed. The wireless chip of WSN node is CC2530 of Texas Instrument company, and each node is equipped with red and green light-emitting diodes to show the node's working status. The distance of the node radio transmission and receiving is about 50 meters. It is especially important that each node is equipped with a flash memory to record the cluster heads number, the rounds number and the cluster head to join battery voltage at every round time. After the experiment is finished, we read the data stored from the flash to analyze. 


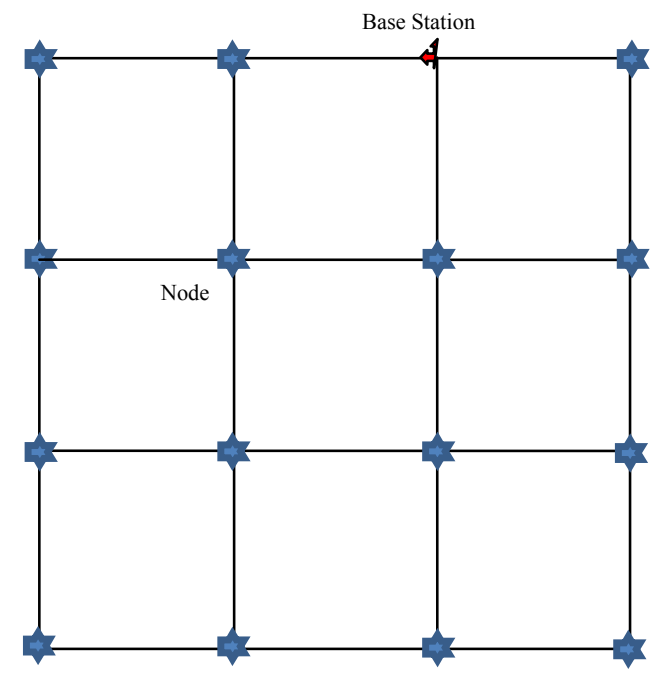

Fig. 3. The nodes location of the actual measurement

We use two algorithms for the practical experiment, the DEBCA proposed in this paper and the LEACH algorithm (the result is shown in Fig. 4).

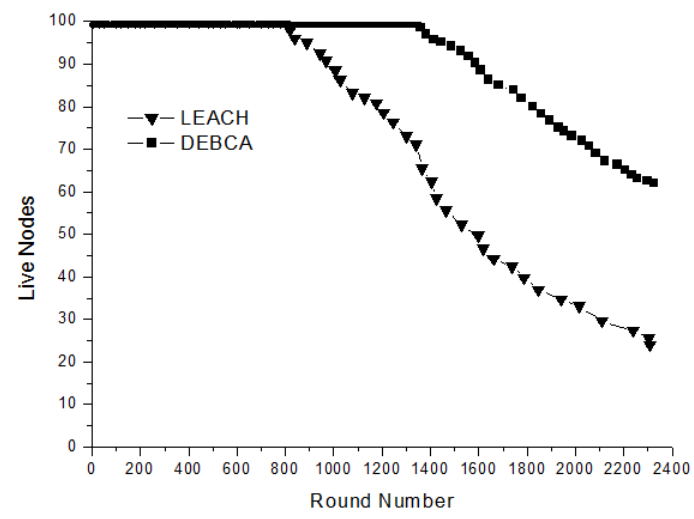

Fig. 4. The live nodes number curve under different algorithms

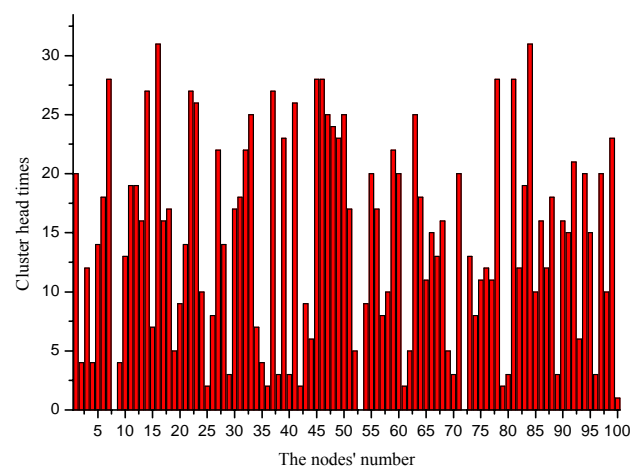

Fig. 5. Nodes as cluster head times in actual measurement 
From this experiment we can see that with the same rounds number - about 850, the number of live nodes with DEBCA is larger than with LEACH in Fig. 4. And the appearance of the first losing nodes in DEBCA comes later than in LEACH for about 600 rounds. Because of the existence of differences among all nodes, including the circuit board itself, the wireless chip, surroundings and batteries, and so on, the energy consuming of each node is different after a long time running. Then we get the flash data from each node, and Fig. 5 shows the times of every node acting as a cluster head. For all nodes, the times when they become a cluster head without average opportunities, satisfy the whole network energy balance rule. Therefore, DEBCA solves the problem of considering the residual energy of all nodes including the cluster head, with the relative distance of each node, which can reduce the vague influence of the application surroundings.

\section{Conclusion}

DEBCA is one kind of a routing protocol developed by LEACH. Unlike LEACH, protocol, DEBCA pays more attention to the residual energy of every node including the cluster head, and every new round the cluster head will attend selecting a new cluster head. In practical applications, the surrounding of each WSNs' node is unknown and may be quite different. After a period of time running, the residual energy of each node will change very much unlike the ideal state; that means the cluster head still may be the node of the cluster with the biggest residual energy. To prolong the lifetime of the network as long as possible, the cluster head with the largest residual energy must be selected for a cluster head again in (2). Under this mechanism, the message load can be reduced greatly during the process of cluster head selecting.

After the new cluster head is selected, when the nodes of the cluster begin to select an appropriate cluster head to join, they must consider the distance factor between the node and the cluster head, and the distance factor between the base station and the cluster head, and the residual energy of the cluster head and the nodes altogether. DEBCA solves the problem in (8), considering all factors in a cluster. In the simulation and actual measurements, DEBCA shows quite improved performance compared to the traditional LEACH. At the same application scene, the appearance of the first losing nodes in DEBCA comes later than in LEACH for about 600 round times, which proves that DEBCA is more suitable for practical applications.

\section{Re e r e n c e s}

1. Shi-Jin, D., J. Xia o-R ong, L. Le-Min. Research and Analysis on Routing Protocols for Wireless Sensor Networks. - In: Proceedings of the IEEE Communications Circuits and Systems, Vol. 1, 2005, No 5, 407-411.

2. A k k a y a, K., M. Y o u n is. A Survey on Routing Protocols for Wireless Sensor Networks. - Ad Hoc Networks, Vol. 3, 2005, No 3, 325-349. 
3. Fabbri, F., C. Buratti, R. Verdone. A Multi-Sink Multi-Hop Wireless Sensor Network Over a Square Region: Connectivity and Energy Consumption Issues. - GLOBECOM Workshops, 2008, IEEE, 1-6.

4. Ji ang Q., D. Manivannan. Routing Protocols for Sensor Networks. - In: 2004 1st IEEE Consumer Communications and Networking Conference, 2004, 93-98.

5. F u k u har a, T., H. I z u m i k a wa, H. I s h i k a wa. Novel Multiple Path Routing Technology for Multi-Hop Wireless Networks. - In: Proceedings of the IEEE Wireless Communications and Networking Conference, Vol. 7, 2004, No 9, 4969-4973.

6. F a n, L., W. Y u. Routing in Vehicular Ad Hoc Networks: A Survey. - IEEE Vehicular Technology Magazine, M. Haahr Etworking Conference, Vol. 2, 2007, No 2, 12-22.

7. He inze $1 \mathrm{man}$, W. R., A. Chandrakas a n, H. B a lakrishnan. Energy-Efficient Communication Protocol for Wireless Microsensor Networks. - In: Proceedings of the Hawaii International Conference on System Sciences, 2000, 223-227.

8. Da ly, E., M. Ha a hr. Social Network Analysis for Routing in Disconnected Delay-Tolerant Manets. - In: ACM MobiHoc, M. Haahr Etworking Conference, 2007, 32-40.

9. S h a, K., J. D u, W. S h i. WEAR: A Balanced, Fault-Tolerant, Energy-Aware Routing Protocol in WSNs. - International Journal of Sensor Networks, Vol. 1, 2006, 156-168.

10. Voulgaris, S., M. Jelasity, M. Van Steen. A Robust and Scalable Peer-to-Peer Gossiping Protocol. - In: Lecture Notes in Computer Science, Vol. 2872, 2005, 47-58.

11. Y u n-Sheng, Y., C. Y i-K ung, L. Y u h-Ching. Energy-Aware QoS Multicast Routing Protocol Based on Genetic Algorithm for the Mobile Ad Hoc Networks. - Journal of Internet Technology, Vol. 7, 2006, No 2, 131-137.

12. X i e, F., L. D u, Y. B a i, L. C h e n. Energy Aware Reliable Routing Protocol for Mobile Ad Hoc Networks.- In: Wireless Communications and Networking Conference, 2007, 4313-4317.

13. S h a n g, Y., W. R u m i, Y. Z h a n g. Localization from Connectivity in Sensor Networks. - IEEE Trans. On Parallel and Distributed Systems, Vol. 3, 2004, No 2, 22-26.

14. De b, B., S. B hat nagar, B. N ath. ReInForM: Reliable Information Forwarding Using Multiple Paths in Sensor Networks. - In: Proceedings 28th Annual IEEE International Conference on Local Computer Networks, LCN'03, 2003, 406-415.

15. Sh i o S ingh, K., M. P. S ingh, D. K. S ingh. A Survey of Energy-Efficient Hierarchical Cluster-Based Routing in Wireless Sensor Networks. - Int. J. of Advanced Networking and Applications, Vol. 2, 2010, No 2, 570-580.

16. He in z e $1 \mathrm{~m}$ a n, W. R., A. Ch a $\mathrm{d} \mathrm{r}$ a k a s a n. Energy-Efficient Communication Protocol for Wireless Microsensor Networks. - In: Proceedings of the 33rd Annual Hawaii International Conference on System Sciences, 2000.

17. Lindsey, S., C. S. Raghavendra. PEGASIS: Power-Efficient Gathering in Sensor Information Systems. - In: Aerospace Conference Proceedings, 2002. IEEE, Vol. 3, 2002, 1125-1130.

18. Manje s hwar, A., D. P. A graw a 1. TEEN: A Routing Protocol for Enhanced Efficiency in Wireless Sensor Networks. IPDPS, 2001.

19. B o u 1 is, A., M. B. S rivas t a va. Node-Level Energy Management for Sensor Networks in the Presence of Multiple Applications. - Wireless Networks, Vol. 10, 2004, No 6, 737-746.

20. A bus a ime h, H., S. H. Y ang. Dynamic Cluster Head for Lifetime Efficiency in WSN. International Journal of Automation and Computing, Vol. 6, 2009, No 1, 48-54.

21. R h e e, S., D. S e e th a r a m, S. Li u. Techniques for Minimizing Power Consumption in Low Data-Rate Wireless Sensor Networks. - In: Wireless Communications and Networking Conference IEEE, 2003, 1727-1731.

22. R a n, G., H. Z h a n g, S. G o n g. Improving on LEACH Protocol of Wireless Sensor Networks Using Fuzzy Logic. - Journal of Information \& Computational Science, Vol. 7, 2010, No 3, 767-775.

23. Heinzelman, W. R., A. P. Chandrakasan, H. Balakrishnan. An ApplicationSpecific Protocol Architecture for Wireless Microsensor Networks [J]. - IEEE Trans. on Wireless Communications, Vol. 1, 2002, No 4, 660-670. 